\title{
Synthesis of ZnO Nanoparticles from Goat Slaughter waste for Environmental Protection
}

\author{
Anal K. Jha' ${ }^{\dagger}$ and K. Prasad ${ }^{\ddagger}{ }^{*}$ \\ Aryabhatta Centre for Nanoscience and Nanotechnology, Aryabhatta Knowledge University, Patna 800 001, India \\ Accepted 15 Jan 2016, Available online 20 Jan 2016, Vol.6, No.1 (Feb 2016)
}

\begin{abstract}
In this study, a novel technique of utilizing effectively the goat slaughter waste is reported with a conviction that dead animals and their tissue and/or organ wastes too can participate in nanoparticle synthesis. This effort may help in controlling the environmental pollution and subsequently the different diseases. A simple environment friendly protocol, employing the broth of goat slaughter waste (principally intestine) yielded $\mathrm{ZnO}$ nanoparticles. X-ray and transmission electron microscopy analyses are performed to ascertain the formation of ZnO nanoparticles. Individual nanoparticles as well as a few aggregates having the size of 3-11 $\mathrm{nm}$ are found. Also, the reaction protocol was monitored using UV-visible spectroscopy. Accordingly, the entire span of experimentation (absorbance-time data) has been proposed in the form of a mathematical model. The surface plasmon resonance was observed at $359 \mathrm{~nm}$. A possible involved mechanism for the biosynthesis of $\mathrm{ZnO}$ nanoparticles has also been proposed.
\end{abstract}

Keywords: Nano ZnO, Nanomaterials, Waste minimization, Green approach, Biosynthesis, Nanobiotechnology, Environmental pollution

\section{Introduction}

We all need food of different kinds to nourish ourselves and to pamper our pallet. Some prefer vegetarian food while others survive on non-vegetarian options comprising of meat from different higher or lower animal sources. The production of meat across the farm to fork continuum produces not just meat for human consumption, but also the processing waste. The nature and quantity of the waste varies at each stage, but includes the carcasses of dead animals, parts of animals which are treated as inedible, bones, hides and blood. The quantity of meat production waste is staggering. Humans consume only a portion of a food animal. A significant portion of food animals become waste. Approximately $50-54 \%$ of each cow, $52 \%$ of each sheep or goat, $60-62 \%$ of each pig, $68-72 \%$ of each chicken and $78 \%$ of each turkey end up as meat consumed by humans with the remainder becoming waste after processing (Regulations 2003). Immediately after evisceration (i.e. removal of inner organs of the body like heart, lung, intestine, kidney etc.) the problem of their disposal surfaces after slaughtering. Slaughter house waste contains mostly biodegradable matter but due to the lack of awareness

*Corresponding author: K. Prasad ${ }^{\dagger}$ Also at University Department of Chemistry, T.M. Bhagalpur University, Bhagalpur 812007, India

${ }^{\ddagger}$ Also at University Department of Physics, T.M. Bhagalpur University, Bhagalpur 812007, India they are not disposed properly and are left to fester, which causes pollution and become wonderful source for disease causing microbes.

So, the problem is not confined to an area or country rather it has gained a dreadful magnitude worldwide. Discards of animals whether in the processing industry or along the road side generates biological wastes principally containing gut and other parts. Though, in India, the poor masses do consume the alimentary canal yet this problem looms quite large. However, quite recently, efforts have been made to produce bio-gas from wash offs of such discards (Ek et al. 2011, Ahmad and Ansari 2012) yet the problem is too big to be handle effectively. Goats are among the earliest animals domesticated by humans. Capra aegagrus hircus (domestic goat) is common specimen which is routinely sacrificed for meat all over India. The waste thus generated is either left to fester or is thrown in water bodies which contaminate it.

Keeping this in mind, we had recently reported the use of lower animals like banana fly (Drosophila sp.) (Jha and Prasad 2012), cockroach (Periplaneta americana) (Jha and Prasad 2013) and fish discard (Jha and Prasad 2014a) broths as model systems with a view to synthesize different nanoparticles using animal dressing/discard being generated after sacrifice of animals. Along with this, as the biological synthetic methods have an added advantage that they are totally green and in-line with RoHS guidelines of the EU, accordingly this work reports the biosynthesis of $\mathrm{ZnO}$ 
nanoparticles using goat slaughter waste (principally intestine) for the first time. Zinc oxide as such is an excellent adsorbent and tends to adsorb biologically generated bad odour which is quite rampant in India. Here, we suggest an effective mean to productively recycle and utilize a biological waste to control the menace of pollution caused principally due to biologically generated odour following a microbe assisted festering.

\section{Experimental details}

\subsection{Preparation of culture medium employing goat intestine waste}

Known quantity (200 gm) goat processing discard (principally gut) was collected from local mutton market with Capra aegagrus hircus as the species of choice for the same. It was washed thoroughly thrice by deionized water in order to get rid of dust or other undesired materials like blood stains along with metallic contamination if any. The discards were immediately ground in the presence of $50 \%$ ethanol, mixed and gently heated until the colour of mixture changes from clear transparent to deep straw indicating dissolution of significant metabolites. This extract was carefully filtered after gently meshing the animal tissues, pooled and cooled for 20 minutes at room temperature. The finely meshed animal tissue was further boiled in fresh pool of sterile distilled water $(200 \mathrm{ml})$ for another 10 minutes. This too was cooled and filtered in order to ensure complete extraction of the probable candidate metabolites. Thereafter, both the extracts were mixed and utilized for the preparation of ZnO-NPs.

\subsection{Biosynthesis of $\mathrm{ZnO}$ nanoparticles}

Analytical reagent grade zinc chloride $\left(\mathrm{ZnCl}_{2}\right)$ was taken into use for preparing a solution of 0.25 (M) strength at room temperature in sterile distilled water. $20 \mathrm{ml}$ of solution was added to the diluted juice (without shifting the $\mathrm{pH}$ beyond 6) and it was heated until appearance of starch like haziness in solution and white deposition at the bottom of flask was noted. This was perceived as initiation of transformation. The value of $\mathrm{pH}$ was maintained at 6 because a lower value delays the procedure of oxide synthesis and a higher value leads to metallization. The flask was allowed to incubate in the laboratory ambience for another four hour till duck white fluffy mass settles down leaving clear transparent supernatant at the top. It was also filtered for further studies.

\subsection{Characterizations}

The formation and crystal structure of $\mathrm{ZnO}$ nanoparticles was ascertained by X-ray diffraction (XRD) technique using an $\mathrm{X}$-ray diffractometer (XPERT-PRO) with $\mathrm{CuK}_{\alpha}$ radiation $\lambda=1.5406 \AA$ over a wide range of Bragg angles $\left(20^{\circ} \leq 2 \theta \leq 75^{\circ}\right)$. TEM micrographs and selected area diffraction (SAED) patters of $\mathrm{ZnO}$ nanoparticles was obtained using Bruker transmission electron microscope. The specimens were suspended in distilled water, dispersed ultrasonically to separate individual particles, and two drops of the suspension deposited onto holey-carbon coated copper grids. The absorption spectrum was obtained by a computer interfaced UVvisible spectrophotometer (Hitachi U-2800, Japan).

\section{Results}

Figure 1 shows the powder XRD pattern obtained for $\mathrm{ZnO}$ nanoparticles synthesized using goat slaughter waste. A number of Bragg reflections $(2 \theta)$ of different lattice planes of wurtzite hexagonal zinc oxide were estimated using the software POWD. The observed reflections were found similar to the observed reflections in bulk $\mathrm{ZnO}$. The lattice parameters: $a=$ $3.253 \AA$ and $a=5.211 \AA$ as obtained, are in excellent agreement with the literature report (ICDD \#: 086254). Besides, the broadening of XRD peaks is primarily due to the small particle size.

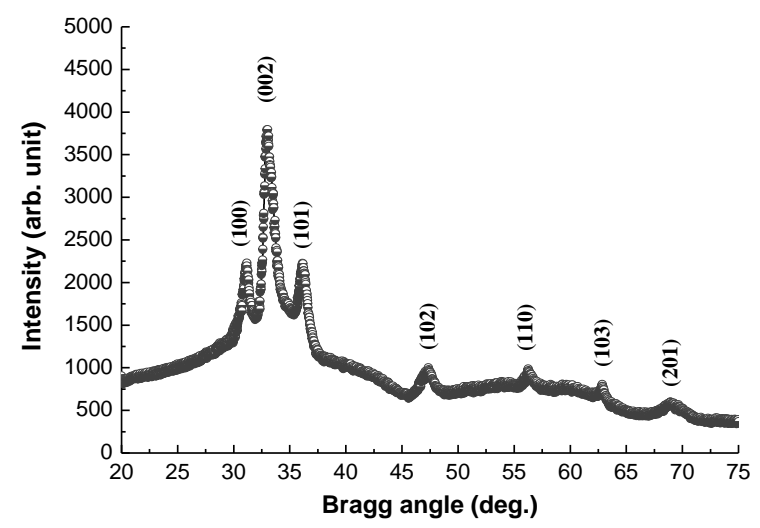

Fig. 1 X-ray diffraction pattern of ZnO NPs at room temperature synthesized using goat slaughter waste broth with $\mathrm{ZnCl}_{2}$ solution

Figure 2 shows the TEM micrograph of $\mathrm{ZnO}$ nanoparticles being formed using goat slaughter waste. The micrograph clearly illustrates individual nanoparticles as well as a few aggregates. The particle size was calculated with the help of ImageJ software considering 50 particles. The particles are found almost spherical in shape having a size of the order of 3-11 $\mathrm{nm}$. The average particle size comes out to be 5.92 $\pm 0.21 \mathrm{~nm}$ after the statistical analysis. The difference in size may possibly be due to the fact that the nanoparticles are being formed at different times. Also, the Scherrer rings as seen in the SAED pattern (inset of Figure 2) with plane distances of 2.789, 2.581 and $2 \cdot 439 \AA$ A corresponding to 100,002 and 101 planes, clearly indicates the formation of pure wurtzite hexagonal $\mathrm{ZnO}$ nanocrystalline particles. This is in good agreement with XRD result. Considering the nanoparticles to be spherical and is made up of $n$ number of unit cells having the edge lengths $a$ and $c$, 
then $\frac{4}{3} \pi R^{3}=n V$, where $V=3 \sqrt{3} / 2 a^{2} c$ (for hexagonal structure) is the volume of the unit cell. Hence, $n=\frac{4}{3} \pi R^{3} / V$. The value of $n$ estimated to be 753 for $\mathrm{ZnO}$ nanoparticles.

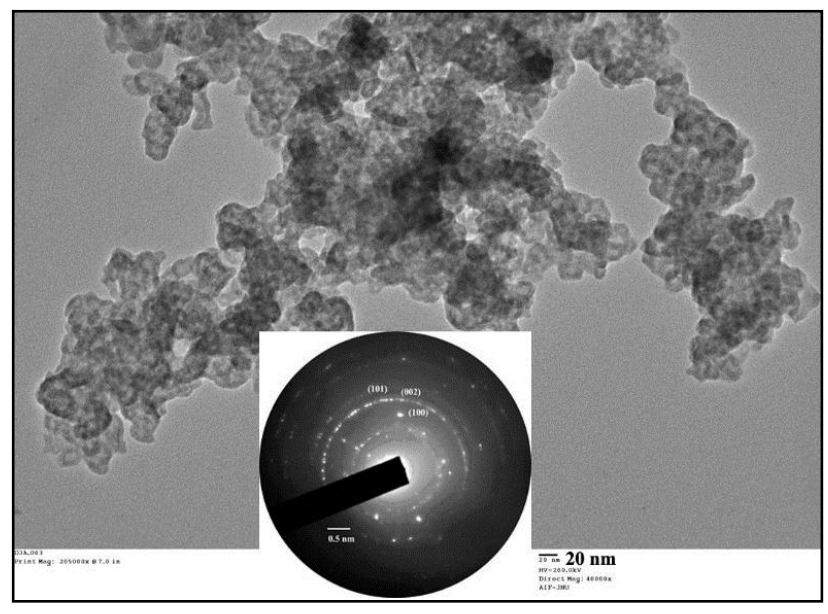

Fig. 2 TEM image of $\mathrm{ZnO}$ NPs synthesized by $\mathrm{Zn}^{2+}$ ions using goat slaughter waste broth. Inset: SAED pattern.

The UV-vis absorption spectrum of the $\mathrm{ZnO}$ nanoparticle was recorded as a function of wavelength in the wavelength range $300-800 \mathrm{~nm}$ as shown in Figure 3. The surface plasmon resonance was observed at $359 \mathrm{~nm}$. Besides, it is observed that the plasmon bands are broadened with an absorption tail in the longer wavelengths, which may be due to the size distribution of the particles (Jha and Prasad 2010, Jha and Prasad 2014b). The inset of Figure 3 depicts the formation of $\mathrm{ZnO}$ nanoparticles taking place at a faster rate and saturation of data occurs at $250 \mathrm{~min}$ which clearly indicates the completion of reaction. The absorbance at $\lambda_{\max }(\xi)$ - time data were modeled with the function of type:

$\xi(t)=\xi_{\mathrm{s}}-\kappa \cdot r^{t}$

where the parameters $\xi_{s}$ and $r(0<r \leq 1)$ are respectively perceived as the saturation value of absorbance and the rate of reaction. $\kappa$ is the material dependent constant, which may be reckoned as the response range of the reaction. It is observed that the experimental data fits excellently well $\left(R^{2}=0.98735\right)$ with the proposed theoretical model (inset Figure 3). The computer fitting of $\xi(t)$ data with Eqn.(1) provided the model parameters: $\xi_{s}=3.1006, \kappa=2.05749$ and $r$ $=0.98869$. The inset of Figure 3 illustrates the reduction of the silver ions taking place at a faster rate and that saturation of data occurs at $250 \mathrm{~min}$ which clearly indicates the completion of reaction. It seems that the present procedure of biosynthesis of $\mathrm{ZnO}$ nanoparticles proceeds with a drastically faster pace and formation of $\mathrm{ZnO}$ nanoparticles is complete in approximately four hours.

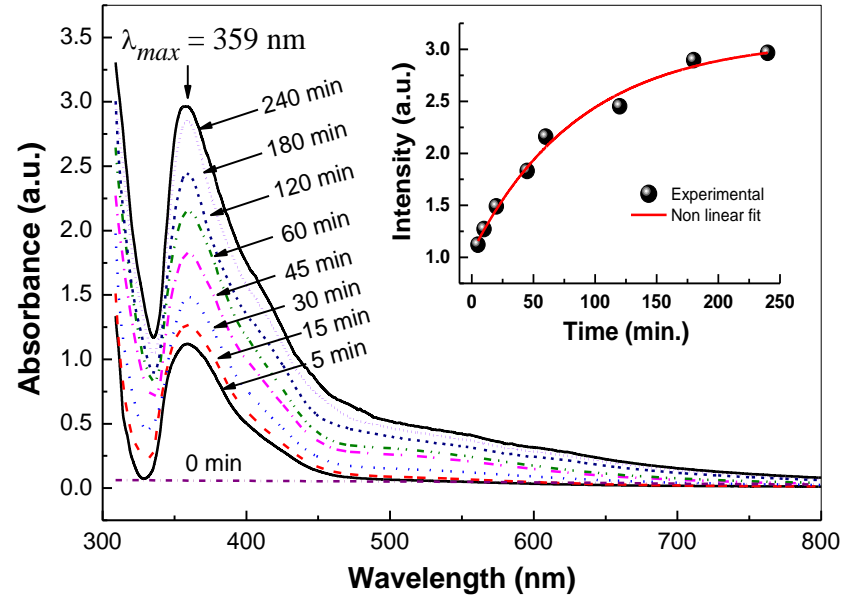

Fig. $3 \mathrm{UV}$-vis spectra of ZnO NPs recorded as function of time of reaction of $0.25 \mathrm{M}$ aqueous solution of $\mathrm{ZnCl}_{2}$ with goat slaughter waste broth. Inset: Absorbance at

$\lambda_{\max }(\xi)$ as a function of time with theoretical fit to Eqn.(1).

It is considered that the nano-sized $\mathrm{ZnO}$ particles should have a wider band gap than the bulk material owing to the quantum confinement of the electronhole pair that forms due to the absorption of a sufficiently energetic photon. The larger energy difference causes a shift in the visible absorbance spectrum of $\mathrm{ZnO}$. Optical excitation of electrons across the band gap is strongly allowed, producing an abrupt increase in absorptivity at the wavelength corresponding to the gap energy. This feature in the optical spectrum is known as the optical absorption edge. The most direct way of extracting the optical band gap is to simply determine the wavelength at which the extrapolations of the base line and the absorption edge meets. The particle radius can be estimated using the relation: $E_{g}=E_{g}^{0}+\hbar^{2} \pi^{2} / 2 \mu R^{2}$, where $E_{g}$ and $E_{g}^{0}$ are the band gap value of the nanoparticles and the bulk material respectively, $\mu=m_{e} m_{h} /\left(m_{e}+m_{h}\right)$ is the reduced mass and $m_{e}$ and $m_{h}$ are effective masses of electrons in conduction-band and holes in valence-band respectively. Here the reduced mass of the exciton was taken to be $0 \cdot 242 m_{0}$ (Singh et al. 2007). The values of $E_{g}$ obtained were $3.46 \mathrm{eV}$, this value is shifted compared with the bulk value (3.37 eV) ( $\mathrm{Zu}$ et al. 1997) and this could be a consequence of a 'size quantization' effect in the samples. Therefore, as expected, reduction in particle size gives a shift in the optical band gap of the samples. The particle radius thus calculated are $4.16 \mathrm{~nm}$, which is in fairly good agreement with TEM result.

\section{Discussion}

Domestic goats (Capra aegagrus hircus) are herbivores and are quite vulnerable to different types of exposures including pesticides, heavy metals etc. The nature has bestowed upon them the suitable 
physiological vigour in order to circumvent such chemical stresses. Their digestive system is quite well developed and ensures their proper nutrition and survival. Although some heavy metals are essential as trace elements, most can be, at high concentrations, toxic to all forms of life, including microbes, humans and animals. Herbivores eat varieties of plants which enrich them with a plethora of promising phytochemicals and keep them healthy.

Effective protection against oxidative damage is crucial for the functioning of organisms. The antioxidant defence systems of living organisms are mainly composed by non-enzymatic antioxidants (e.g. glutathione, ascorbic acid, $\alpha$-tocopherol and $\beta$ carotene) and specific antioxidant enzymes. The major antioxidant enzymes are superoxide dismutases (SOD), glutathione peroxidases (GPx) and catalase (CAT) that are located in different cellular compartments in virtually all tissues of vertebrates, although showing in general, higher activities in liver (Cnubben et al. 2001). The alimentary canal of goats is principally made up of proteins and is equipped with weaponry for detoxification. MTs have a high heavy metal affinity and binding capacity (7-9 g atom mol-1thionein) and are able to chelate both essential (e.g. zinc and copper) and non-essential metals (e.g. cadmium and mercury) by cysteine tetrathiolate clusters. The metal-thiolate clusters form in two well-defined domains, named $\beta$ (N-terminal) and $\alpha$ (C-terminal), with stoichiometry of M3Cys9 and M4Cys11, respectively, for divalent metal ions (Vašák et al. 2005). The metallotetrathiolate clusters provide the protein with a highly stable tertiary structure that renders it stability to heat (Kägi et al. 1987). The metal-thiolate clusters within the MT molecules allow rapid exchanges of metallic ions between clusters and with other molecules. These characteristics of binding and transference of metals appear to be unique to MT and fundamental to their biological role (Viarengo et al. 2000).

However, it should be noted that MT could protect the cells from oxidative stress not only acting as oxyradical scavenger, but through metal binding/release dynamics (Viarengo et al. 2000). Mammalian species have multiple MT genes coding for a family of isoforms. In fact, distinct MT isoforms designated MT-1 through MT-4 has been found (Vašák and Hasler 2000, Milles et al. 2000). MT-1 and MT-2 isoforms are present in all organs being the best studied. MT-3 is expressed mainly in brain and MT-4 is most abundant in certain stratified tissues (like intestine). It is now established that from insects to mammals, along with others, metallothionein genes are induced in response to heavy metal load by the transcription factor MTF-1, which bind to short DNA sequence motifs, termed metal response elements (MREs). Remarkable earlier works, (Anfinsen 1946, Bailey 1948), established the idea that de-naturation of soluble proteins involved transitions from a relatively compact orderly structure to a more flexible, disorganized, open polypeptide chain. More than 65 years after its initial discovery (Bailey 1946, Bailey 1946), the actin binding, coiled- coil protein tropomyosin (Tm) (Bailey 1948) is a fascinating biological molecule whose structural, stability, and functional properties remain incompletely understood. However, Tm is expressed in all eukaryotic cell types in more than 40 isoforms that are widely distributed within individual cells (Perry 2001, Gunning et al. 2008). As has been apparent for many years from a range of experimental methods, including DSC, in terms of thermodynamic free energy, folded proteins are only marginally stable with respect to their unfolded states. Since all molecules are always in perpetual thermal motion (and thermodynamics is merely a consequence of this) it is useful to bear in mind the average thermal energies involved in such motion. Classical statistical thermodynamics (Equipartition theorem) show that every independent form of motion or degree of freedom in a molecule has a mean thermal energy of $1 / 2 k_{\mathrm{B}} T$, where $\mathrm{k}_{\mathrm{B}}$ is Boltzmann's constant and $T$ is the absolute temperature. For kinetic energy or translational motions there are three degrees of freedom, corresponding to movement along $x y z$ axes, so average kinetic energy is $3 / 2 k_{\mathrm{B}} T$. Similarly for free rotational motion the average energy will be about $1 / 2 k_{\mathrm{B}} T$ per rotational degree of freedom. Vibrational modes have two degrees of freedom each - one translational and one extensional - but for covalent bonds at least the classical equipartition approximation breaks down. Quantization of vibrational levels has to be considered here and conventional bond vibrations are rarely excited at normal temperatures. A useful rule of thumb is that the average thermal energy associated with each motional degree of freedom in a molecule is of order $k_{\mathrm{B}} T$ per molecule, or RT per mole. This corresponds to about $2.5 \mathrm{~kJ} \mathrm{~mol}^{-1}\left(0.6 \mathrm{kcal} \mathrm{mol}^{-1}\right)$ at room temperature. Many experimental methods for estimating thermodynamic parameters for protein transitions rely on the assumption of 2-state behaviour for the system. In the case of proteins, A and B might be the native (N) and unfolded (U) states, respectively, and the transition may be brought about by changes in temperature, $\mathrm{pH}$ or denaturant concentration.

The experimental free energy difference ( $\Delta G$ unf) between folded and unfolded states under nearphysiological conditions is usually in the range $+20-60$ $\mathrm{kJ} \mathrm{mol}{ }^{-1}$. Experimentally, it has been found that the temperature dependence of $\Delta G$ unf shows that for most proteins the folded form is, not unreasonably perhaps, most stable in the physiological temperature range. The relatively small free energy of unfolding is made up of, usually, much larger and much more temperature dependent enthalpy and entropy contributions (Cooper 1999). In the light of above mentioned facts, we may emphatically perceive that even in case of dead animal tissues, its pivotal molecules do not remain in-effective rather they are thermodynamically agile to liberate or absorb energy. This liberated energy probably ensures the phase transformation in the present case from microscale to the nanoscale. 


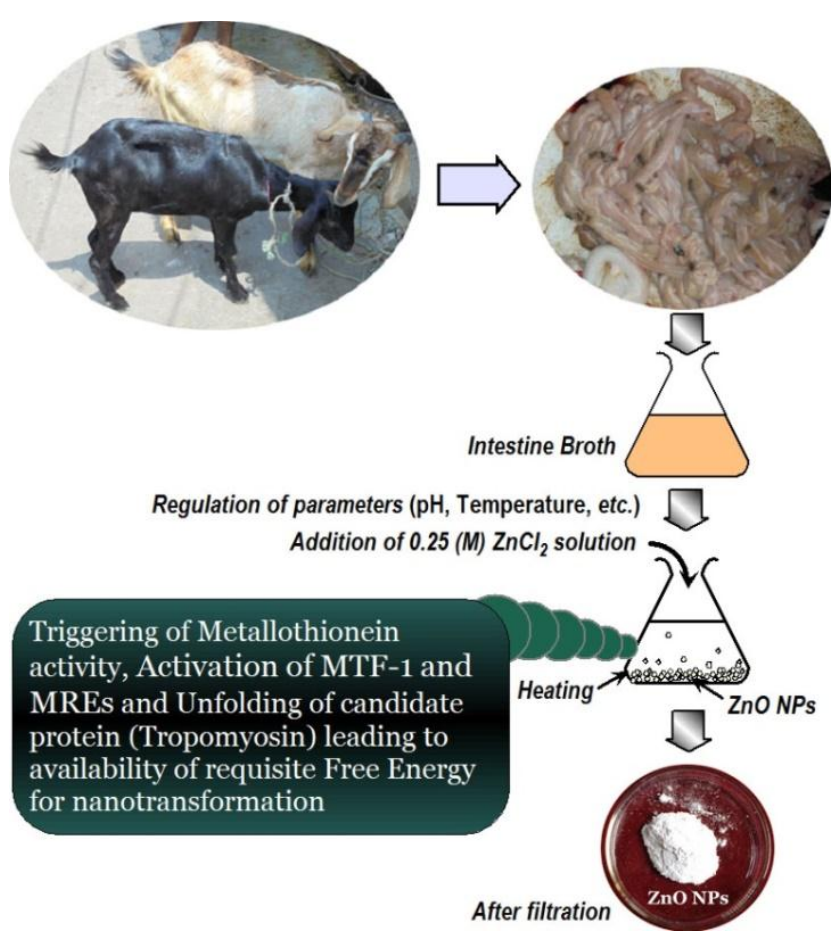

Fig. 4 Mechanism of biosynthesis of $\mathrm{ZnO}$ nanoparticles using goat slaughter waste broth

Discards of goat principally in the form of intestine; being taken into use for preparing ZnO NPs, in this maiden effort; still harbour promising metabolites which are otherwise wasted by festering. Though, the cells die after boiling but the liberated metabolites from the tissues help in nanomaterial fabrication. The probable bio-synthetic mechanism is detailed in Figure 4. Earlier by applying this idea, we had reported synthesis of metal and chalcogenide nanoparticles using dead cockroach and banana fly broths, fish discard and the present effort consolidates our conviction that the discards of animals can effectively contribute towards synthesis of nanoparticles. Our effort in utilising the discard of other animal processing is still on.

\section{Conclusions}

In summary, ZnO NPs have successfully been prepared using goat slaughter waste (principally intestine). Therefore, present biosynthetic method is capable of producing $\mathrm{ZnO}$ nanoparticles, which might have resulted due to the activity of metallothioneine, indicated thereby that animal's discard too can negotiate nano-transformations. Furthermore, present work might be a step towards utilizing animal system and/or animal discard in synthesizing a variety of nanomaterials and consequently addressing environmental issues.

\section{References}

Ahmad, J., Ansari, T.A., (2012), Biogas from slaughterhouse waste: Towards energy self-sufficient industry with economical analysis in India, J. Microbial. Biochem. Technol., S12, 1-4.

Anfinsen, C.B., (1973), Principles that govern the folding of protein chains, Science, 181, 223-230.

Anfinsen, C.B., and Scheraga, H.A., (1975), Experimental and theoretical aspects of protein folding, Adv. Protein Chem., 29, 205-300.

Bailey, K., (1946), Tropomyosin: a new asymmetric protein of muscle, Nature, $157,368$.

Bailey, K., (1948), Tropomyosin: a new asymmetric protein of the muscle fibril, Biochem. J., 43, 271-279.

Cooper, A., (1999), Thermodynamics of Protein Folding and Stability, In: Geoffrey, A. (Editor), Protein: A comprehensive treatise. JAI Press Inc., USA, 2, 217-270.

Cnubben, N.H.P., Rietjens, I.C., Wortelboer, H., van Zanden, J., and van Bladeren, P.J., (2001), The interplay of glutathione-related processes in antioxidant defense, Environ. Toxicol. Pharmacol., $10,141-152$.

Edsall, J.T., (1995), Hsien Wu and the first theory of protein denaturation, Adv. Protein Chem., 46, 1-5.

Ek, A.E.W., Hallin, S., Vallin, L., Schnürer, A., and Karlsson, M., (2011), Slaughterhouse waste co-digestion - Experiences from 15 years of full-scale operation, Bioenergy Technology, World Renewable Energy Congress, Linkoping, Sweden, p. 64-71.

Gunning, P., O'Neill, G., and Hardeman, E., (2008), Tropomyosinbased regulation of the actin cytoskeleton in time and space, Physiol. Rev., 88, 1-35.

Jha, A.K., Prasad, K., (2010), Green synthesis of silver nanoparticles using Cycas leaf, Inter. J. Green Nanotechnol: Phys. Chem., 1, 110-117.

Jha, A.K., Prasad, K., (2012), Banana fly (Drosophila Sp.) synthesizes CdS nanoparticles!, J. Bionanosci., 6, 99-103.

Jha, A.K., Prasad, K., (2013), Can animals too negotiate nano transformations?, Adv. Nano Res., 1, 35-42.

Jha, A.K., Prasad, K., (2014a), Synthesis of silver nanoparticles employing fish processing discard: an eco-amenable approach, J. Chin. Adv. Mater. Soc., 2, 179-185.

Jha, A.K., Prasad, K., (2014b), Green synthesis of silver nanoparticles and its activity on SiHa cervical cancer cell line, Adv. Mater. Lett. 2014, 5, 501-505

Kägi, J.H.R., and Kojima, Y., (1987), Chemistry and biochemistry of metallothionein, Experientia Suppl., 52, 25-61.

Milles, A.T., Hawksworth, G.M., Beattie, J.H., and Rodilla, V. (2000), Induction, regulation, degradation, and biological significance of mammalian metallothioneins, Critical Rev. Biochem. Mol. Biol., 35, 35-70.

Perry, S.V., (2001), Vertebrate tropomyosin: distribution, properties and function, J. Muscle Res. Cell Motil., 22, 5-49.

Singh, P., Chawla, A.K., Kaur, D., and Chandra R., (2007), Effect of oxygen partial pressure on the structural and optical properties of sputter deposited $\mathrm{ZnO}$ nanocrystalline thin films, Mater. Lett., 61, 2050-2053.

The Animal By-products (Scotland) Regulations 2003, Training Seminar Materials (Edinburgh, 4 November 2003); EU, Questions and Answers on Animal By-Products (Brussels, 6 May 2004), available from http://europa.eu.int/comm/dgs/health_consumer/library/pr ess/press152_en.pdf.

Vašák, M., (2005), Advances in metallothionein structure and functions, J. Trace Elements Med. Biol., 19, 13-17.

Vašák, M., Hasler, and D.W., (2000), Metallothioneins-new structural and functional insights, Curr. Opinion Chem. Biol., 4, 177-183.

Viarengo, A., Burlando, B., Ceratto, N., and Panfoli, I., (2000), Antioxidant role of metallothionein: a comparative overview Cell Molecular Biol., 46, 407-417.

Zu. P., Tang, Z.K., Wong, G.K.L., Kawasaki, M., Ohtomo, A., Koinuma, H., and Segawa, Y., (1997), Ultraviolet spontaneous and stimulated emission from $\mathrm{ZnO}$ microcrystallites at room temperature, Solid State Commun., 103, 459-463. 